\title{
BMJ Open Atherogenic dyslipidaemia and cardiovascular events in patients with diabetes or pre-diabetes and stable coronary artery disease: a prospective, cohort study
}

\author{
Jing-Lu Jin, ${ }^{1,2}$ Ye-Xuan Cao, ${ }^{1}$ Li-Guo Wu, ${ }^{3}$ Xiang-Dong You, ${ }^{3} \mathrm{Na}$ Guo, ${ }^{3}$ \\ Yuan-Lin Guo, ${ }^{1}$ Na-Qiong Wu, ${ }^{1}$ Cheng-Gang Zhu, ${ }^{1}$ Rui-Xia Xu, ${ }^{1}$ Hui-Hui Liu, ${ }^{1}$ \\ Jing Sun, ${ }^{1}$ Qian Dong, ${ }^{1}$ Ying Gao, ${ }^{1}$ Jian-Jun Li (D) ${ }^{1}$
}

To cite: Jin J-L, Cao Y-X, Wu L-G, et al. Atherogenic dyslipidaemia and cardiovascular events in patients with diabetes or prediabetes and stable coronary artery disease: a prospective, cohort study. BMJ Open 2021;11:e037340. doi:10.1136/ bmjopen-2020-037340

- Prepublication history and additional material for this paper are available online. To view these files, please visit the journal online (http://dx.doi. org/10.1136/bmjopen-2020037340).

Received 30 January 2020 Revised 08 December 2020 Accepted 09 December 2020

D) Check for updates

(C) Author(s) (or their employer(s)) 2021. Re-use permitted under CC BY-NC. No commercial re-use. See rights and permissions. Published by BMJ.

For numbered affiliations see end of article.

\section{Correspondence to}

Professor Jian-Jun Li; lijianjun938@126.com and Dr Ying Gao; cmuxyg@sina.com

\section{ABSTRACT}

Objective The aim of the study was to investigate the impacts of triglyceride (TG) and high-density lipoprotein cholesterol (HDL-C) dyslipidaemia on prognosis in coronary artery disease (CAD) patients with different glucose metabolism status.

Design An observational cohort study.

Setting/participants A total of 3057 patients with stable CAD were consecutively enrolled and divided into three groups according to different glucose metabolism status. Atherogenic dyslipidaemia (AD) was defined as $T G \geq 1.7$ $\mathrm{mmol} / \mathrm{L}$ and $\mathrm{HDL}-\mathrm{C}<1.0 \mathrm{mmol} / \mathrm{L}$ for men or $<1.3 \mathrm{mmol} / \mathrm{L}$ for women. The patients were further classified into six subgroups by status of $A D$. All subjects were followed up for the cardiovascular events (CVEs).

Primary outcome measures The primary endpoints were cardiovascular mortality, non-fatal myocardial infarction and non-fatal stroke.

Results During a median follow-up of 6.1 years, 308 (10.1\%) CVEs occurred. No significant difference in the occurrence of CVEs was observed between normal glucose regulation (NGR) and pre-diabetes (pre-DM) groups (HR: $1.25,95 \% \mathrm{Cl} 0.89$ to 1.76 ) while DM group presented 1.45-fold higher risk of CVEs (HR: $1.45,95 \% \mathrm{Cl} 1.02$ to 2.05). When the participants were categorised according to combined status of two parameters, the cardiovascular risk was significantly elevated in pre-DM or DM plus $A D$ group compared with the NGR plus non-AD group (HR: $1.76,95 \% \mathrm{Cl} 1.10$ to 2.80 and $\mathrm{HR}: 1.87,95 \% \mathrm{Cl} 1.17$ to 2.98).

Conclusions The present study suggested that the presence of $A D$ might affect the prognosis in patients with $\mathrm{DM}$ or pre-DM and stable CAD.

\section{INTRODUCTION}

Dyslipidaemia is one of the key drivers in atherogenesis. Lipid lowering therapy targeting at low-density lipoprotein cholesterol (LDL-C) has been proved to be efficient in secondary prevention of arteriosclerotic cardiovascular disease (ASCVD). ${ }^{1}$ Moreover,
Strengths and limitations of this study

- This study fills the gap of the current knowledge on the predictive value of atherogenic dyslipidaemia in patients with coronary artery diseases and impaired glucose metabolism.

- The study focuses on hard endpoints during a relatively long follow-up period, which might provide reliable information concerning the impact of dyslipidaemia on outcomes in such patients.

- This is a single centre, observational study among Chinese patients with stable coronary artery disease (CAD).

- For inevitable reasons, this study is restricted to the predictive value of baseline parameters.

- More studies may be necessary in different kinds of population such as unstable patients with $C A D$ and subjects in randomised clinical trials.

strong evidence from epidemiological, genetic and prospective cohort studies verifies that high triglyceride (TG) and/or low levels of high-density lipoprotein cholesterol (HDL-C) are associated with cardiovascular disease (CVD) risk. $^{2-4}$ It has been demonstrated in large-scale clinical trials that hypertriglyceridaemia was associated with increased cardiovascular events (CVEs). ${ }^{5}$ However, clinical trials about therapeutic interventions in patients afflicted with low HDL-C did not show convincing results. Anacetrapib reduced CVEs by $9 \%$ in the Randomised Evaluation of the Effects of Anacetrapib through Lipid-modification study but it was not clear whether the risk reduction was attributed to the increase in HDL-C. ${ }^{6}$ Type 2 diabetes mellitus (T2DM) is also one of the major risk factors of CVD. ${ }^{7}$ Atherogenic dyslipidaemia $(\mathrm{AD})$, defined as low HDL-C accompanied 
with elevated TG, is one of the most important comorbidities in T2DM. Previous studies indicated that individuals with $\mathrm{AD}$ presented higher risk of CVD in patients with DM. ${ }^{8}$ In the Action to Control Cardiovascular Risk in Diabetes (ACCORD) trial, patients with a combination of high baseline TG $(\geq 204 \mathrm{mg} / \mathrm{dL}$, highest tertile) and low baseline HDL-C ( $\leq 34 \mathrm{mg} / \mathrm{dL}$, lowest tertile) showed possible benefit from fenofibrate plus simvastatin therapy compared with simvastatin alone. ${ }^{9}$ Standards of medical care in diabetes from American Diabetes Association (ADA) also recommended intensify lifestyle therapy and optimise glycaemic control for patients with elevated TG levels and/or low HDL cholesterol. ${ }^{10}$

Pre-diabetes (pre-DM) is an abnormal glucose regulation status with high predisposition to developing T2DM. Pre-DM subjects had similar lipid profile as patients with DM. However, the prognosis of patients with pre-DM with coronary artery disease $(\mathrm{CAD})$ was rarely estimated. Also, evidence about whether the pre-DM alone or accompanied by $\mathrm{AD}$ can increase CVD risk in patients with $\mathrm{CAD}$ is lacking. The aim of the study is to test the hypothesis that pre-DM and DM plus AD had significant impacts on cardiovascular outcomes in patients with angiographyproven CAD.

\section{MATERIALS AND METHODS}

\section{Study design and participants}

From March 2011 to November 2013, 4249 consecutive patients were scheduled for coronary angiography because of clinically suspected CAD. Among these patients, 413 were excluded because they did not meet the diagnostic criteria of CAD (with a stenosis more than $50 \%$ of the at least one major coronary artery). Other exclusion criteria were described in the flowchart (figure 1). As reported in detail previously, ${ }^{11}{ }^{12}$ patients were followed up for primary endpoints which included cardiovascular mortality, non-fatal myocardial infarction (MI) and stroke.

DM and pre-DM were diagnosed according to according to ADA criteria. ${ }^{13}$ Patients who were without DM or pre-DM were defined as normal glucose regulation (NGR, fasting plasma glucose $<5.6$ and haemoglobin A1c (HbA1c) level $<5.7 \%$ ). AD was defined as TG $\geq 1.7$ $\mathrm{mmol} / \mathrm{L}$ and HDL-C $<1.0 \mathrm{mmol} / \mathrm{L}$ for men or $<1.3$ $\mathrm{mmol} / \mathrm{L}$ for women. Hypertension was defined as a selfreported hypertension, currently taking antihypertensive drugs or recorded systolic blood pressure (SBP) $\geq 140 \mathrm{~mm}$ $\mathrm{Hg}$ and/or diastolic blood pressure (DBP) $\geq 90 \mathrm{~mm} \mathrm{Hg}$ for three or more consecutive times. Information of other disease, family history and prior therapy of every patient was also documented.

\section{Laboratory analysis}

Blood samples were obtained from each patient from the cubital vein after at least 12 hours fasting. Concentrations of total cholesterol (TC), TG, LDL-C, HDL-C were measured using automatic biochemistry analyser (Hitachi

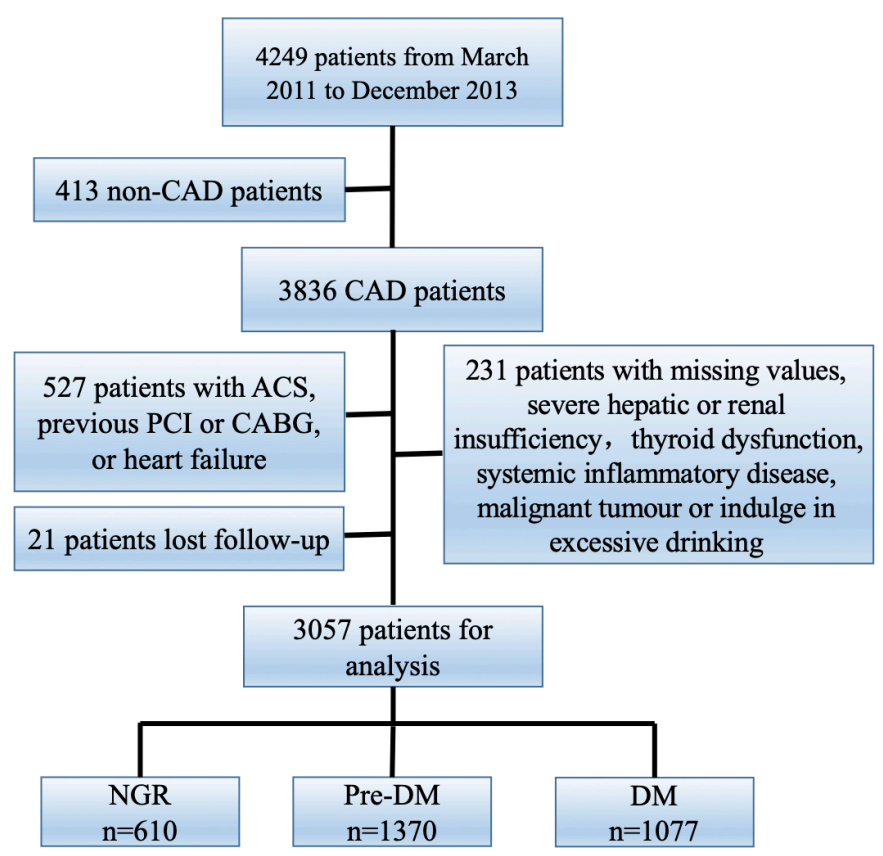

Figure 1 Flowchart of the study. ACS, acute coronary syndrome; CABG, coronary artery bypass grafting; $C A D$, coronary artery disease; DM, diabetes mellitus; NGR, normal glucose regulation; $\mathrm{PCl}$, percutaneous coronary intervention; pre-DM, pre-diabetes mellitus.

7150, Tokyo, Japan) in an enzymatic assay. The concentrations of glucose were measured by enzymatic hexokinase method. HbAlc was measured using Tosoh Automated Glycohemoglobin Analyser (HLC-723G8, Tokyo, Japan).

\section{Evaluation of coronary severity}

Angiographic data were evaluated from catheter laboratory records by three experienced interventional cardiologists according to our previous studies. ${ }^{14}$ The Gensini score (GS) was calculated as previously described. ${ }^{15}$

\section{Statistical analysis}

The values were expressed as the mean $\pm \mathrm{SD}$ or median (Q1-Q3 quartiles) for the continuous variables and the number (percentage) for the categorical variables. The Kolmogorov-Smirnov test was used to test the distribution pattern. The differences of clinical characteristics between groups were analysed using the Student's t-test, analysis of variance or non-parametric test, $\chi^{2}$ statistic test or Fisher exact test where appropriate. The eventfree survival rates among groups were estimated by the Kaplan-Meier method and compared by the log-rank test. Univariate and multivariate Cox regression analyses were performed to calculate the HRs. Adjust variables were traditional cardiovascular risk factors including age, sex, body mass index (BMI), smoking, hypertension, family history of CAD, GS, left ventricular ejection fraction (LVEF), LDL-C, HDL-C, TG, high sensitive $\mathrm{C}$ reactive protein and baseline statins. A $p$ value $<0.05$ was considered statistically significant. The statistical analyses were performed with SPSS V.21.0 software. 


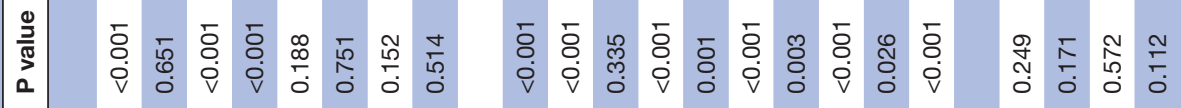

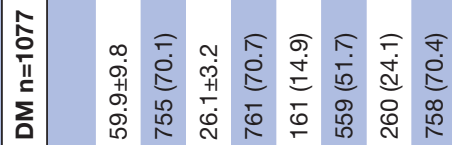

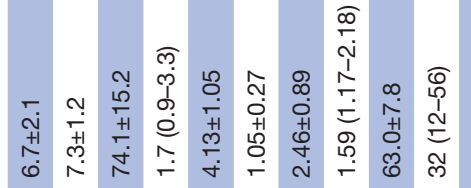
$\begin{array}{llll}0 & 0 \\ 0 & 0 & 0 & 0 \\ 0 & 0 & 0 & 0 \\ 0 & 0\end{array}$ ๑

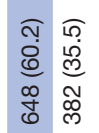

a n 

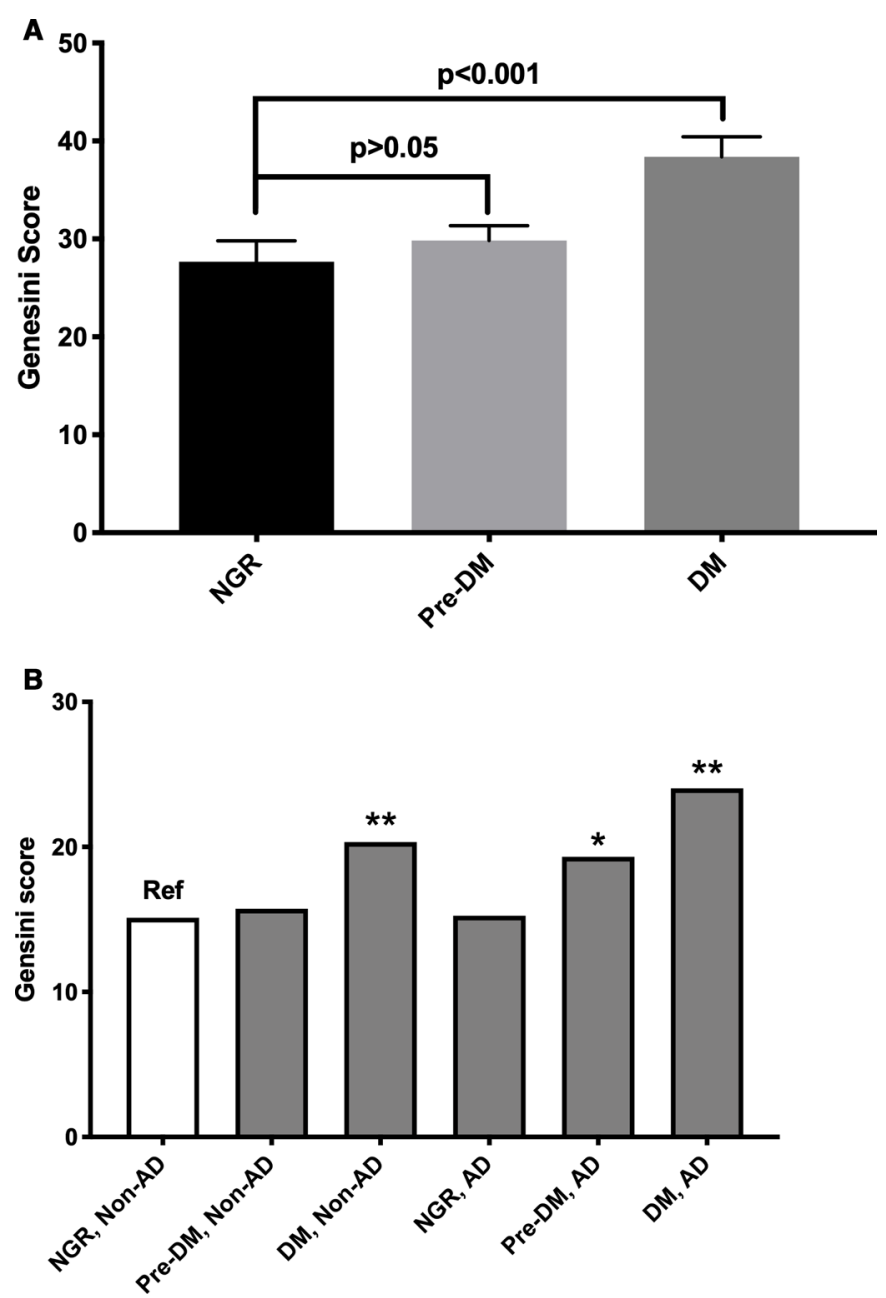

Figure 2 Coronary severity in participants according to (A) different glucose metabolism; (B) different status of glucose metabolism and atherogenic dyslipidaemia. ${ }^{*} \mathrm{p}<0.05$; ${ }^{\star *} p<0.01$. AD, atherogenic dyslipidaemia; DM, diabetes mellitus; NGR: normal glucose regulation.

\section{Patient and public involvement}

Patients or the public were not involved in the design, or conduct, or reporting, or dissemination plans of our research.

\section{RESULTS}

As presented in figure 1, 20.0\%, $44.8 \%$ and $35.2 \%$ of 3057 subjects were diagnosed as NGR, pre-DM and DM, respectively according to ADA criteria. The baseline characteristics of the study participants were shown in table 1 . The age, BMI, glucose, HbA1c, TG and high-sensitivity C reactive protein (hsCRP) and proportion of hypertension were elevated from NGR to DM (all $\mathrm{p}<0.001$ ). Patients with pre-DM and DM had elevated levels of TC and LDL-C than the NGR group. Meanwhile, NGR patients had significantly higher levels of HDL-C and LVEF than DM population. There was no significant difference regarding other demographic and laboratory parameters among the three groups $(\mathrm{p}>0.05)$.
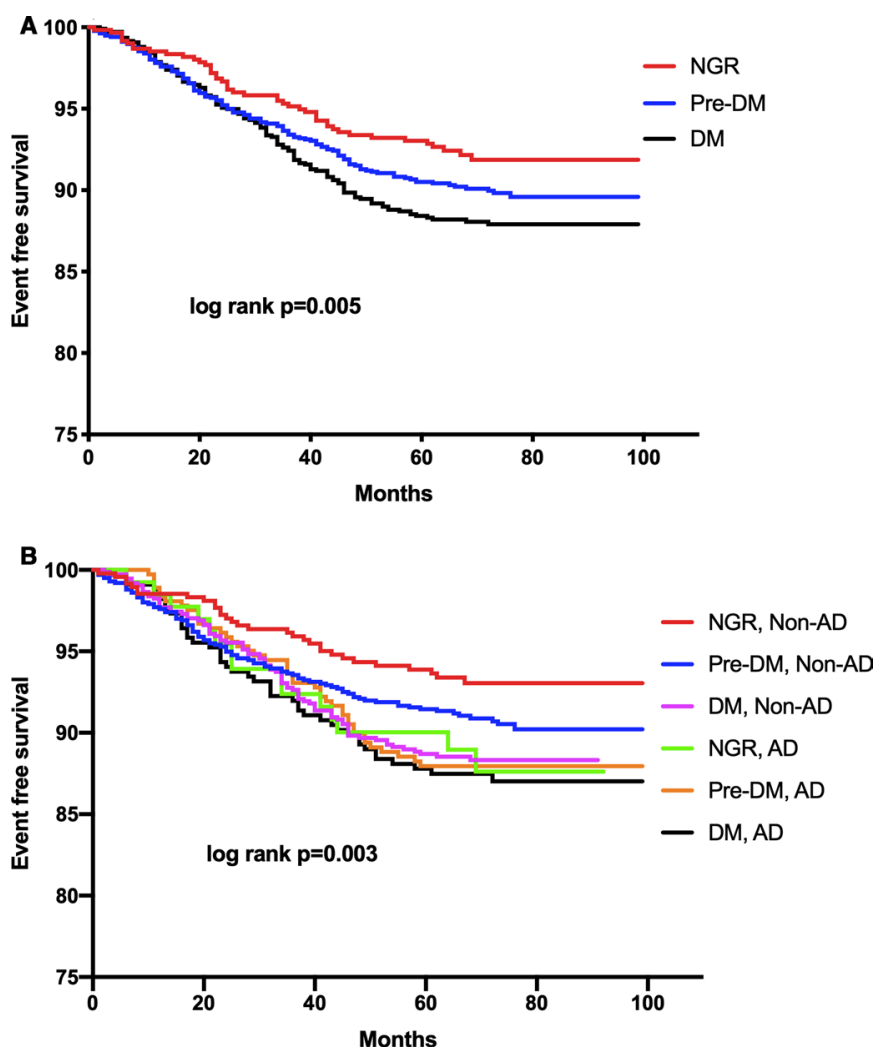

Figure 3 Kaplan-Meier curves and log-rank tests for cardiovascular events in participants according to (A) different glucose metabolism; (B) different status of glucose metabolism and atherogenic dyslipidaemia. $A D$, atherogenic dyslipidaemia; DM, diabetes mellitus; NGR, normal glucose regulation.

The coronary severity was compared among different status of glucose metabolism. As shown in figure 2A,B, DM group had significantly higher GS $(p<0.05)$ while there was no significant difference between pre-DM and NGR groups $(p>0.05)$. We further divided the patients into the six groups according to status of glucose metabolism and AD (NGR plus non-AD; pre-DM plus non-AD; $\mathrm{DM}$ plus non-AD; NGR plus $\mathrm{AD}$; pre-DM plus $\mathrm{AD}$; $\mathrm{DM}$ plus $\mathrm{AD}$ ). We set NGR and non-AD group as reference and compared its GS with that of other groups. All the other groups had higher GS than the reference group (all $\mathrm{p}<0.05$ ) except pre-DM plus non-AD and NGR plus $\mathrm{AD}$ group ( $>0.05$, respectively). As shown in online supplemental table $\mathrm{S} 1$, multivariate regression logistic regression analysis revealed that DM group was independently associated with high GS (median as cut-off, $\mathrm{p}<0.05$ ). Pre-DM plus AD group and DM plus AD group were also independently associated with the presence of high GS (all $\mathrm{p}<0.05$, online supplemental table S2).

Over a median follow-up time of 6.1 years (5.1-7.5 years), 308 CVEs occurred, including 112 cardiovascular deaths, 73 non-fatal MI and 123 had non-fatal strokes. 7.5\%, $9.9 \%$ and $11.8 \%$ of patients had CVEs in NGR, pre-DM and DM groups, respectively. As indicated in KaplanMeier analysis (figure 3A), DM subjects had the highest event rate among the three groups $(\mathrm{p}<0.05)$ while there 
Table 3 Cox regression models in predicting cardiovascular events according to different status of glucose metabolism and atherogenic dyslipidaemia

\begin{tabular}{|c|c|c|c|}
\hline \multirow[b]{3}{*}{ DM/AD category } & \multicolumn{3}{|l|}{ HR (95\% Cl) } \\
\hline & Events/subjects & & \\
\hline & $308 / 3057$ & Crude model & Adjusted model \\
\hline Pre-DM, non-AD & $92 / 1005$ & 1.42 (0.94 to 2.13$)$ & 1.40 (0.92 to 2.10$)$ \\
\hline$D M$, non-AD & $84 / 741$ & *1.75 (1.16 to 2.64$)$ & *1.68 (1.11 to 2.56$)$ \\
\hline NGR, AD & $15 / 133$ & 1.74 (0.94 to 3.22$)$ & 1.74 (0.94 to 3.23$)$ \\
\hline $\mathrm{DM}, \mathrm{AD}$ & $43 / 336$ & *1.95 (1.23-3.09) & *1.87 (1.17-2.98) \\
\hline
\end{tabular}

Model adjusted for age, sex, body mass index, smoking, hypertension, family history of coronary artery disease, Gensini score, left ventricular ejection fraction, low-density lipoprotein cholesterol, high-sensitive $\mathrm{C}$ reactive protein and baseline statins.

${ }^{*} \mathrm{p}<0.05$.

$A D$, atherogenic dyslipidaemia; DM, diabetes mellitus; NGR, normal glucose regulation; pre-DM, pre-diabetes mellitus.

was no significant difference between that of pre-DM and NGR groups $(\mathrm{p}>0.05)$. However, when the patients were evaluated according to both glucose metabolism and $\mathrm{AD}$ status: $\mathrm{DM}$ plus non-AD, pre-DM plus $\mathrm{AD}$ and $\mathrm{DM}$ plus $\mathrm{AD}$ groups had significantly lower cumulative event-free survival rates compared with the reference group (NGR plus non-AD group, figure $3 \mathrm{~B}$, all $\mathrm{p}<0.05$, respectively). As presented in table 2, univariate Cox regression models showed that patients with DM had 1.45-fold higher risk of CVEs than NGR subjects (HR: 1.45, 95\% CI 1.02 to 2.05 , $\mathrm{p}<0.05$ ). The GS was also associated with CVEs (HR: 1.004, 95\% CI 1.001 to $1.008, \mathrm{p}<0.05]$. Additional adjustment for confounding variables including GS did not change the significance of association. The presence of pre-DM did not show increase in CVEs risk when compared with NGR group ( $p>0.05)$. Moreover, multivariate Cox regression analyses according to both glucose metabolism and $\mathrm{AD}$ status indicated that patients in $\mathrm{DM}$ plus non- $\mathrm{AD}$, pre-DM plus $\mathrm{AD}$ and $\mathrm{DM}$ plus $\mathrm{AD}$ groups had 1.68-fold (95\% CI 1.11 to 2.56 ), 1.76 -fold (95\% CI 1.10 to 2.80 ) and 1.87 -fold (95\% CI 1.17 to 2.98) higher risk of CVEs (table 3 , all $\mathrm{p}<0.05$, respectively).

\section{DISCUSSION}

The relation of high TG and/or low HDL-C to ASCVD risk has been controversial during the past decades. Previous prospective studies have shown that patients with high TG combined with low HDL-C may be more likely to develop
ASCVD, especially in those with DM. ${ }^{16}$ In this study, we investigated the impact of $\mathrm{AD}$ on cardiovascular outcomes in stable, angiography-proven patients with $\mathrm{CAD}$ with different glucose metabolism status. We found that patients with DM but not those with pre-DM had more severe coronary stenosis and higher risk of CVEs when the patients were simply divided into the three groups: DM, pre-DM and NGR. Interestingly, when patients were categorised according to both status of glucose metabolism and $\mathrm{AD}$, individuals with pre-DM plus $\mathrm{AD}$ had higher GS and 1.76-fold increased risk of CVEs than NGR and non-AD subjects. Thus, our study suggested that the presence of $\mathrm{AD}$ may have an impact on cardiovascular outcomes in patients with CAD and DM or pre-DM.

High TG and low HDL-C are common lipid abnormalities among adult population, especially in Chinese subjects. According to DYSlipidemia International Study, 41.8\% patients had high TG and $31.9 \%$ patients had low HDL-C among Chinese population. ${ }^{17}$ Additionally, studies about reducing CVD risk by lowering TG and raising HDL-C had inconsistent results. ${ }^{69}$ For example, fibrates did not have conclusive effect in ASCVD risk reduction in ACCORD trials while patients who received $2 \mathrm{~g}$ of icosapent ethyl two times per day had lower risk of ischaemic events in Reduction of Cardiovascular Events with Icosapent Ethyl-Intervention Trial. ${ }^{918}$ However, in randomised controlled trials, cholesteryl ester transfer protein inhibitors, which could increase plasma HDL-C, failed to reduce CVEs rates. ${ }^{919-21}$ In the meanwhile, Mendelian analysis involving about $20000 \mathrm{MI}$ individuals and

Table 2 Cox regression models in predicting cardiovascular events according to different glucose metabolism

\begin{tabular}{llll}
\hline \multirow{2}{*}{ Diabetic status (n, events/subjects) } & HR (95\% Cl) & Model $\mathbf{1}$ & Model 2 \\
\cline { 2 - 4 } NGR (46/610) & Unadjusted model & Ref & Ref \\
\hline Pre-DM (135/1379) & Ref & $1.29(0.92$ to 1.81$)$ & 1.25 (0.89 to 1.76$)$ \\
DM (127/1077) & $* 1.56(1.11$ to 2.19$)$ & $* 1.53(1.09$ to 2.15$)$ & $* 1.45(1.02$ to 2.05$)$ \\
\hline
\end{tabular}

Model 1 adjusted for age and sex; model 2 adjusted for age, sex, body mass index, smoking, hypertension, family history of coronary artery disease, Gensini score, left ventricular ejection fraction, low-density lipoprotein cholesterol, high-density lipoprotein cholesterol, triglyceride, high sensitive $\mathrm{C}$ reactive protein and baseline statins. ${ }^{*} \mathrm{p}<0.05$.

DM, diabetes mellitus; NGR, normal glucose regulation; pre-DM, pre-diabetes mellitus. 
50000 controls demonstrated that $1 \mathrm{SD}$ increase in TG levels was associated with $54 \%$ increased risk of $\mathrm{MI}^{22}$ In contrast, no such association was found for patients with low baseline levels of HDL-C. ${ }^{3}$ Moreover, in the EPIC-Norfolk prospective population study, healthy men with $\mathrm{AD}$ had $61 \%$ higher risk of CAD than those with normal TG and HDL-C. ${ }^{23}$ Of noted, patients who were with obesity, insulin resistance or other metabolic abnormalities had higher prevalence of high TG and/or low HDL-C. ${ }^{24}$

DM was the most common metabolic disease in the 21st century. Approximately 415 million adults were with T2DM worldwide. ${ }^{25}$ Prevalence of total diagnosed and undiagnosed diabetes in China reached $10.9 \%$ in $2013{ }^{26}$ What is more, CAD was a common comorbidity in patients with DM. According to previous studies, patients with DM without angiography-proven CAD showed low risk of MI or CVEs (defined as death, cardiac death and $\mathrm{MI}$ ), but the DM and CAD combination further increased the risk of ischaemic stroke. ${ }^{27}{ }^{28}$ In our previous studies, among patients with established CAD, individuals with DM were associated with significantly higher risk of worse prognosis when they were combined with other $\mathrm{CAD}$ risk factors, including hypertension and $\mathrm{Lp}(\mathrm{a})$ hyperlipoproteinaemia. ${ }^{14}{ }^{29}$ Therefore, in the present study, among patients with stable CAD under different glucose metabolism status, identifying whether $\mathrm{AD}$ is a risk factor for worse prognosis might be crucial. In strong heart study, high TG plus low HDL was associated with a 1.54-fold greater occurrence risk for CAD and a 2.13-fold occurrence risk for stroke in community based African Americans with DM. ${ }^{8}$ In a large cohort of 28318 DM subjects, increased CAD risk was observed in both men and women with $\mathrm{AD} .^{16}$ In the ACCORD trial, for participants with DM, fenofibrate plus simvastatin $40 \mathrm{mg}$ exhibited a $31 \%$ reduction in CVEs in the subgroup with baseline high TG and low HDL-C. ${ }^{9}$

In fact, more attention has recently been paid to the clinical characters in the early phase of impaired glucose metabolism for the prevention of DM. Pre-DM was an intermediate state between NGR and DM and with high predisposition to develop DM. This metabolic condition was often reversible. The rate of individuals with pre-DM was almost three times higher than that of DM worldwide and in China ( $35.7 \%$ vs $10.9 \%$ in China).$^{25}{ }^{30}$ The prevalence of pre-DM and CVEs risk have long been debating. There were different cut-off points in the various definitions to diagnose pre-DM. Studies and meta-analysis using similar blood glucose and HbAlc cut-offs according to 2003 ADA guideline also had different results. ${ }^{31} 32$ In our study population, as previously reported, the predictive value of pre-DM for CVEs was less significant, which was also consistent with studies conducted by Liu et al and Qiu et al. ${ }^{143}$ In the present study, $21.8 \%, 26.6 \%$ and $31.2 \%$ of patients had AD in NGR, pre-DM and DM groups. Both pre-DM and DM groups had higher rate of AD than NGR group. As the main findings of our study, stable patients with $\mathrm{CAD}$ and pre-DM plus $\mathrm{AD}$ had higher GS and increased risk of CVEs while no statistically significant difference were observed between pre-DM plus non-AD and NGR plus non-AD groups. Therefore, similar attention should be given to patients with pre-DM and DM when they were with $\mathrm{AD}$.

The present study had several virtues compared with previous published reports. Very few studies have evaluated the differences of coronary severity and outcomes according to both status of glucose metabolism and $\mathrm{AD}$, especially in those with stable CAD. In addition, previous studies were also limited by the fact that the risk of high TG and/or low HDL-C levels were analysed separately within DM or NGR population, neglecting of the potential high risk which was caused by the interaction of lipid and glucose. Moreover, there were no such studies about the impacts of $\mathrm{AD}$ on cardiovascular outcomes in patients with CAD and pre-DM. Apparently, a large sample size of angiography-proven patients with $\mathrm{CAD}$ with high prevalence of DM and pre-DM were enrolled in the present study. Hard endpoints containing non-fatal strokes, nonfatal MI, and cardiovascular mortality were also observed during a relatively long follow-up period. Thereby, our study provided important information regarding dyslipidaemia, glucose metabolism status and outcome.

Nevertheless, there are still several limitations in the present study. First, this is a single centre study among Chinese patients with stable CAD. Second, we measured TG, HDL-C and glucose metabolism status only at the baseline. The follow-up levels of TG/HDL-C may also be clinically significant. According to previous study, during the follow-up period, a small proportion of subjects with pre-DM may develop DM each year. ${ }^{34}$ The increased CAD severity and CVEs may be overestimated in the pre-DM group. Third, we did not assess all metabolic factors and parameters about insulin resistance due to the features of patients in our study. Fourth, even if AD plus NGR group did not present increased CVEs risk, there is possibility that the result missed the statistical significance level due to smaller number of subjects. Hence, further studies with larger sample size may be needed.

In conclusion, in our large sample size with long-term follow-up study, data indicated that the patients with pre-DM and DM with $\mathrm{AD}$ had significantly higher risk of CVEs, suggesting that treatment and lifestyle management towards $\mathrm{AD}$ in patients with pre-DM and $\mathrm{DM}$ may also be crucial for improving clinical outcomes.

\section{Author affiliations}

${ }^{1}$ State Key Laboratory of Cardiovascular Disease, Fu Wai Hospital, National Center for Cardiovascular Diseases, Chinese Academy of Medical Sciences, Peking Union Medical College, BeiLiShi Road 167,100037, Beijing, China

${ }^{2}$ Department of Endocrinology, Genetics and Metabolism, Beijing Children's Hospital, Capital Medical University, National Center for Children's Health, 100045, Beijing, China

${ }^{3}$ Department of cardiology, TangXian Peoples Hospital, Baoding, China

Contributors Study concept and design: J-JL, YG and J-LJ. Acquisition of data: J-JL, Y-X C and J-LJ. Analysis and interpretation of data: YG and J-LJ. Drafting of the manuscript: J-LJ. Critical revision of the manuscript for important intellectual content: J-JL. Statistical analysis: J-LJ. Obtained funding: J-JL and YG. 
Administrative, technical or material support: L-GW, X-DY, Y-LG, N-QW, C-GZ, H-HL, R-XX, JS and QD. Study supervision: J-JL.

Funding This work was partially supported by the Capital Health Development Fund (201614035), CAMS Major Collaborative Innovation Project (2016-I2M-1-011), Fundamental Research Funds for the Central Universities (2018-F05) and Youth Research Fund of Peking Union Medical College (2018-XHQN03).

Competing interests None declared.

Patient consent for publication Not required.

Ethics approval The study was performed according to the Declaration of Helsinki, and the hospital ethics review board (Fuwai Hospital and National Center for Cardiovascular Diseases, Beijing, China) approved the protocol.

Provenance and peer review Not commissioned; externally peer reviewed.

Data availability statement Data are available upon reasonable request. The technical appendix, statistical code and data set are available from the corresponding author.

Supplemental material This content has been supplied by the author(s). It has not been vetted by BMJ Publishing Group Limited (BMJ) and may not have been peer-reviewed. Any opinions or recommendations discussed are solely those of the author(s) and are not endorsed by BMJ. BMJ disclaims all liability and responsibility arising from any reliance placed on the content. Where the content includes any translated material, BMJ does not warrant the accuracy and reliability of the translations (including but not limited to local regulations, clinical guidelines, terminology, drug names and drug dosages), and is not responsible for any error and/or omissions arising from translation and adaptation or otherwise.

Open access This is an open access article distributed in accordance with the Creative Commons Attribution Non Commercial (CC BY-NC 4.0) license, which permits others to distribute, remix, adapt, build upon this work non-commercially, and license their derivative works on different terms, provided the original work is properly cited, appropriate credit is given, any changes made indicated, and the use is non-commercial. See: http://creativecommons.org/licenses/by-nc/4.0/.

\section{ORCID iD}

Jian-Jun Li http://orcid.org/0000-0003-2536-4364

\section{REFERENCES}

1 Collins R, Reith C, Emberson J, et al. Interpretation of the evidence for the efficacy and safety of statin therapy. Lancet 2016;388:2532-61.

2 Sarwar N, Danesh J, Eiriksdottir G, et al. Triglycerides and the risk of coronary heart disease: 10,158 incident cases among 262,525 participants in 29 Western prospective studies. Circulation 2007; 115:450-8.

3 Khera AV, Kathiresan S. Genetics of coronary artery disease: discovery, biology and clinical translation. Nat Rev Genet 2017;18:331-44.

4 Alexander DD, Miller PE, Van Elswyk ME, et al. A meta-analysis of randomized controlled trials and prospective cohort studies of eicosapentaenoic and docosahexaenoic long-chain omega-3 fatty acids and coronary heart disease risk. Mayo Clin Proc 2017;92:15-29.

5 Miller M, Cannon CP, Murphy SA, et al. Impact of triglyceride levels beyond low-density lipoprotein cholesterol after acute coronary syndrome in the prove IT-TIMI 22 trial. J Am Coll Cardiol 2008;51:724-30.

6 , Bowman L, Hopewell JC, et al. Effects of anacetrapib in patients with atherosclerotic vascular disease. $N$ Engl J Med 2017;377:1217-27.

7 Fox CS, Golden SH, Anderson C, et al. Update on prevention of cardiovascular disease in adults with type 2 diabetes mellitus in light of recent evidence: a scientific statement from the American heart association and the American diabetes association. Circulation 2015;132:691-718.

8 Lee JS, Chang P-Y, Zhang Y, et al. Triglyceride and HDL-C dyslipidemia and risks of coronary heart disease and ischemic stroke by glycemic dysregulation status: the strong heart study. Diabetes Care 2017;40:529-37.

9 ACCORD Study Group, Ginsberg HN, Elam MB, et al. Effects of combination lipid therapy in type 2 diabetes mellitus. N Engl J Med 2010;362:1563-74.
10 American Diabetes Association. 6. Glycemic Targets: Standards of Medical Care in Diabetes-2018. Diabetes Care 2018;41:S55-64.

11 Jin J-L, Sun D, Cao Y-X, et al. Triglyceride glucose and haemoglobin glycation index for predicting outcomes in diabetes patients with new-onset, stable coronary artery disease: a nested case-control study. Ann Med 2018;50:576-86.

12 Jin J-L, Cao Y-X, Liu H-H, et al. Impact of free fatty acids on prognosis in coronary artery disease patients under different glucose metabolism status. Cardiovasc Diabetol 2019;18:134.

13 American Diabetes Association. Diagnosis and classification of diabetes mellitus. Diabetes Care 2011;34:S62-9.

14 Liu H-H, Cao Y-X, Li S, et al. Impacts of prediabetes mellitus alone or plus hypertension on the coronary severity and cardiovascular outcomes. Hypertension 2018;71:1039-46.

15 Gensini GG. A more meaningful scoring system for determining the severity of coronary heart disease. Am J Cardiol 1983;51:606.

16 Rana JS, Liu JY, Moffet HH, et al. Metabolic Dyslipidemia and Risk of Coronary Heart Disease in 28,318 Adults With Diabetes Mellitus and Low-Density Lipoprotein Cholesterol $<100 \mathrm{mg} / \mathrm{dl}$. Am J Cardiol 2015;116:1700-4.

17 Zhao S, Wang Y, Mu Y, et al. Prevalence of dyslipidaemia in patients treated with lipid-lowering agents in China: results of the dyslipidemia International study (DYSIS). Atherosclerosis 2014;235:463-9.

18 Bhatt DL, Steg PG, Miller M, et al. Cardiovascular risk reduction with Icosapent ethyl for hypertriglyceridemia. $N$ Engl J Med 2019;380:11-22.

19 Barter PJ, Caulfield M, Eriksson M, et al. Effects of torcetrapib in patients at high risk for coronary events. N Engl $\mathrm{J}$ Med 2007;357:2109-22.

20 Schwartz GG, Olsson AG, Abt M, et al. Effects of dalcetrapib in patients with a recent acute coronary syndrome. N Engl J Med 2012;367:2089-99.

21 Lincoff AM, Nicholls SJ, Riesmeyer JS, et al. Evacetrapib and cardiovascular outcomes in high-risk vascular disease. N Engl J Med 2017;376:1933-42.

22 Musunuru K, Kathiresan S. Surprises from genetic analyses of lipid risk factors for atherosclerosis. Circ Res 2016;118:579-85.

23 Rana JS, Visser ME, Arsenault BJ, et al. Metabolic dyslipidemia and risk of future coronary heart disease in apparently healthy men and women: the EPIC-Norfolk prospective population study. Int J Cardiol 2010;143:399-404.

24 Adiels M, Olofsson S-O, Taskinen M-R, et al. Overproduction of very low-density lipoproteins is the hallmark of the dyslipidemia in the metabolic syndrome. Arterioscler Thromb Vasc Biol 2008;28:1225-36.

25 Cho NH, Shaw JE, Karuranga S, et al. IDF diabetes atlas: global estimates of diabetes prevalence for 2017 and projections for 2045. Diabetes Res Clin Pract 2018;138:271-81.

26 Wang L, Gao P, Zhang M, et al. Prevalence and ethnic pattern of diabetes and prediabetes in China in 2013. JAMA 2017;317:2515-23.

27 Olesen KKW, Madsen M, Gyldenkerne C, et al. Diabetes mellitus is associated with increased risk of ischemic stroke in patients with and without coronary artery disease. Stroke 2019;50:3347-54.

28 Olesen KKW, Madsen M, Egholm G, et al. Patients with diabetes without significant angiographic coronary artery disease have the same risk of myocardial infarction as patients without diabetes in a real-world population receiving appropriate prophylactic treatment. Diabetes Care 2017;40:1103-10.

29 Jin J-L, Cao Y-X, Zhang H-W, et al. Lipoprotein(a) and Cardiovascular Outcomes in Patients With Coronary Artery Disease and Prediabetes or Diabetes. Diabetes Care 2019;42:1312-8.

30 Menke A, Casagrande S, Geiss L, et al. Prevalence of and trends in diabetes among adults in the United States, 1988-2012. JAMA 2015;314:1021-9.

31 Laukkanen JA, Mäkikallio TH, Ronkainen K, et al. Impaired fasting plasma glucose and type 2 diabetes are related to the risk of out-ofhospital sudden cardiac death and all-cause mortality. Diabetes Care 2013;36:1166-71.

32 Rijkelijkhuizen JM, Nijpels G, Heine RJ, et al. High risk of cardiovascular mortality in individuals with impaired fasting glucose is explained by conversion to diabetes: the Hoorn study. Diabetes Care 2007:30:332-6.

33 Qiu M, Shen W, Song X, et al. Effects of prediabetes mellitus alone or plus hypertension on subsequent occurrence of cardiovascular disease and diabetes mellitus: Iongitudinal study. Hypertension 2015;65:525-30.

34 Knowler WC, Barrett-Connor E, Fowler SE, et al. Reduction in the incidence of type 2 diabetes with lifestyle intervention or metformin. N Engl J Med 2002;346:393-403. 